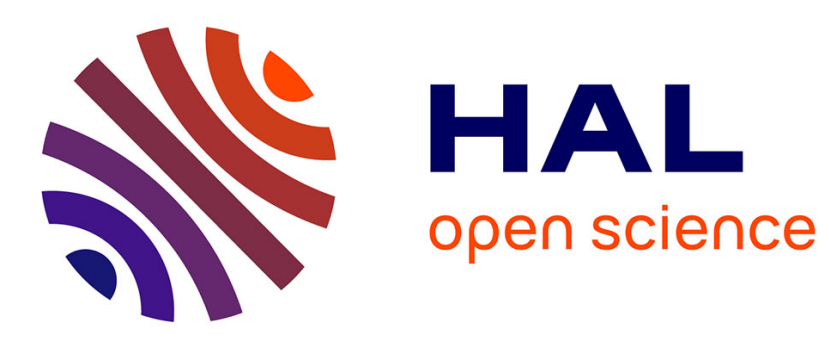

\title{
Comprehensive analysis of chemokine-induced cAMP-inhibitory responses using a real-time luminescent biosensor
}

Virginia Felouzis, Patricia Hermand, Guy Trambly de Laissardière, Christophe Combadière, Philippe Deterre

\section{To cite this version:}

Virginia Felouzis, Patricia Hermand, Guy Trambly de Laissardière, Christophe Combadière, Philippe Deterre. Comprehensive analysis of chemokine-induced cAMP-inhibitory responses using a real-time luminescent biosensor. Cellular Signalling, 2015, 28 (1), pp.120-129. 10.1016/j.cellsig.2015.10.011 . inserm-01229057

\section{HAL Id: inserm-01229057 https://www.hal.inserm.fr/inserm-01229057}

Submitted on 16 Nov 2015

HAL is a multi-disciplinary open access archive for the deposit and dissemination of scientific research documents, whether they are published or not. The documents may come from teaching and research institutions in France or abroad, or from public or private research centers.
L'archive ouverte pluridisciplinaire HAL, est destinée au dépôt et à la diffusion de documents scientifiques de niveau recherche, publiés ou non, émanant des établissements d'enseignement et de recherche français ou étrangers, des laboratoires publics ou privés. 


\section{Comprehensive analysis of chemokine-induced cAMP-inhibitory responses using a real-time luminescent biosensor}

\section{Virginia Felouzis ${ }^{a}$, Patricia Hermand ${ }^{a}$, Guy Trambly de Laissardière ${ }^{b}$,}

Christophe Combadière $^{a}$, Philippe Deterre ${ }^{a}$

a Sorbonne Universités, UPMC Université Paris 06, Inserm U1135, CNRS ERL8255, Centre d'Immunologie et des Maladies Infectieuses, 91 boulevard de l'Hôpital F-75013, Paris, France

b Université de Cergy-Pontoise, CNRS UMR8089, Laboratoire de Physique Théorique et Modélisation, 2 avenue A. Chauvin, F-95302, Cergy-Pontoise, France

Highlights (Include 3 to 5 highlights. There should be a maximum of 85 characters, including spaces, per highlight)

- cAMP-inhibitory responses to chemokines are rarely described in real time observation

- The luminescent biosensor allows monitoring chemokine responses in native conditions

- The chemokine response outline proves to be robust and conserved in different contexts

- This response outline is governed by the chemokine receptor internalization

- The responses to different chemokines exhibit no cross-desensitization

Corresponding author: Philippe Deterre, Centre d'Immunologie et des Maladies Infectieuses, Inserm U1135, Faculté de Médecine. P. et M. Curie, Site Pitié-Salpêtrière, 91 boulevard de 1’Hôpital F-75013, Paris, France. E-mail : philippe.deterre@upmc.fr

Keywords: chemokine, cAMP, biosensor, adenylate cyclase, Gi, forskolin

Abbreviations used: AC, adenylate cyclase; APC, allophycocyanin; AUC, area under curve; CHK, chemokine; EPAC, Exchange Protein directly Activated by cAMP; FSK, forskolin; HEKgloCX3CR1, HEK293 clone stably expressing CX3CR1 and the GloSensor ${ }^{\mathrm{TM}}$ F-22; PDE, phosphodiesterase; $\mathrm{PE}$, phycoerythrin; $\mathrm{PGE}_{2}$, prostaglandin $\mathrm{E}_{2}$; $\mathrm{PKA}$, protein kinase $\mathrm{A}$ 


\begin{abstract}
Chemokine receptors are members of the G-protein-coupled receptor (GPCR) family coupled to members of the Gi class, whose primary function is to inhibit the cellular adenylate cyclases. We used a cAMP-related and PKA-based luminescent biosensor (GloSensor ${ }^{\mathrm{TM}} \mathrm{F}-22$ ) to monitor the real-time downstream response of chemokine receptors, especially CX3CR1 and CXCR4, after activation with their cognate ligands CX3CL1 and CXCL12. We found that the amplitudes and kinetic profiles of the chemokine responses were conserved in various cell types and were independent of the nature and concentration of the molecules used for cAMP prestimulation, including either the adenylate cyclase activator forskolin or ligands mediating Gs-mediated responses like prostaglandin E2 or beta-adrenergic agonist. This tool allowed showing that the cAMP-inhibitory responses to multiple chemokines exhibited no cross-desensitization. Moreover the response kinetics appeared to be governed by the chemokine receptor internalization. Finally, we show that this conserved chemokine response can be accounted for by a simple model only combining Gi inhibitory action on adenylate cyclase and the associated phosphodiesterase activity. We conclude that the cAMP chemokine response is robustly conserved in various inflammatory conditions. Moreover, the cAMP-related luminescent biosensor appear as a valuable tool to analyze the details of Gi-mediated cAMP-inhibitory cellular responses, even in native conditions and could help to decipher their precise role in cell function.
\end{abstract}




\section{Introduction}

Chemokines (CHKs) are secreted, soluble molecules expressed by numerous immune cell types, either constitutively or induced under inflammatory conditions. They play a central role in cell recruitment and positioning in the immune system and during development. CHK signaling is crucial to the inflammatory process and is dysregulated in autoimmune disorders, rheumatologic diseases, and atherosclerosis $[1,2]$. CHK receptors are members of the G-protein-coupled receptor (GPCR) family, specifically the rhodopsin class, coupled to the Gi class of G-proteins. However, some CHK receptors were recently found coupled to the Gq class [3]. The Gi-mediated cAMPinhibitory responses to $\mathrm{CHK}$ were evidenced a long time ago, as early as calcium responses [4]; however, their functional role remains controversial. Some studies have indicated a regulatory role for the cAMP messenger in monocyte chemotaxis towards CCL2 [5], while others have suggested a positive role [6] or no role at all [7]. Therefore, the precise real-time mechanism of CHK-triggered cAMP signaling is still unknown.

Recent use of FRET biosensors [8-12] has unraveled subtle kinetic information about cAMP cellular signaling and the unexpected compartmentation and diffusional restrictions of cAMP and its signaling partners [13-15]. Most of these studies were done with cAMP-stimulatory ligands, such as adrenergic molecules in cardiomyocytes [15] or dopaminergic neuronal responses [16], and rarely addressed the Gi-mediated cAMP-inhibitory signals, such as those evoked by CHKs.

In this study, we investigated the CHK-induced cAMP-inhibitory responses downstream of

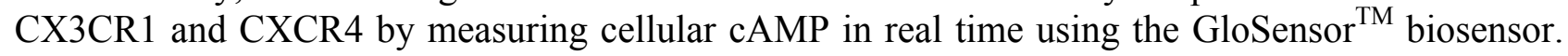
We found that the response kinetics were remarkably conserved with all modes of cAMP prestimulation (forskolin and Gs-associated ligands) and that the period between the additions of the pre-stimulatory ligand and $\mathrm{CHK}$ did not affect the response, indicating the robustness of $\mathrm{CHK}$ action in diverse inflammatory contexts.

\section{Material and methods}

\subsection{Reagents}

Cell culture media was purchased from Gibco, Life Technologies. FBS was obtained from Dutscher Scientific (Les Ulis, France). Forskolin (FSK), 3-isobutyl-1-methylxanthine (IBMX), prostaglandin E2 (PGE2), hygromycin B, and geneticin (G418) were from Sigma-Aldrich. Pertussis toxin was purchased from Enzo Life Sciences. Dynasore was obtained from Calbiochem (Merck Millipore; Guyancourt, France). The 96-well clear-bottomed white microplates were from Greiner-Bio-One (Dutscher Scientific; Les Ulis, France). JetPEI was purchased from Ozyme (Montigny-le Bretonneux, France). The Dual-Luciferase ${ }^{\circledR}$ reporter assay system, pGloSensor $^{\text {TM }}$-22F-cAMP plasmid, and GloSensor ${ }^{\mathrm{TM}}$ cAMP reagent were purchased from Promega (Charbonnière, France). The CX3CL1 CHK domain and CX3CL1 full-length protein were from R\&D Systems (Lille, France), and CXCL12, CXCL26, and CCL2 were from Peprotech (Neuilly sur Seine, France). Mouse monoclonal antibodies against human CX3CR1 and CXCR4 conjugated with allophycocyanin (APC) or phycoerythrin (PE) and IgG1and IgG2B isotype controls were from R\&D Systems (Lille, France). Sp-8-br-cAMP was from BioLog Life Science Institute (Bremen, Germany)

\subsection{Cell culture}

HEK293 cells were cultured in Dulbecco's modified Eagle's medium (DMEM) plus GlutaMAX I supplemented with $10 \%$ FBS, $1 \mathrm{mM}$ sodium pyruvate and $1 \%$ penicillin and streptomycin. Human HEK293 cells expressing the human CX3CR1 gene [18]were transfected with a plasmid encoding an engineered cAMP-sensitive luciferase gene (pGloSensortm-22F-cAMP plasmid, Promega) using the jetPEI method (Polyplus, Ozyme). A stable clone (called HEKgloCX3CR1) was selected for their ability to respond to FSK. The HEKgloCX3CR1 cells were grown in supplemented DMEM as 
above plus geneticin $(0.5 \mathrm{mg} / \mathrm{mL})$ and hygromycin $(0.1 \mathrm{mg} / \mathrm{mL})$.

HBP-ALL and Jurkat cell lines were maintained in Roswell Park Memorial Institute medium (RPMI-1640) supplemented with 10\% FBS. A stable HPB-ALL clone expressing CX3CR1 was made, as previously described [17]. Prior to electroporation, the cells were washed with phosphatebuffered saline (PBS), counted, and resuspended in RPMI-1640 FBS-free media at a cell density of $5 \times 10^{6}$ cells $/ 400 \mu \mathrm{L}$ in a $4 \mathrm{~mm}$ Gene Pulser ${ }^{\circledR}$ cuvette (Bio-Rad; Hercules, CA) and mixed with 10 $\mu \mathrm{g}$ of the pGloSensor ${ }^{\mathrm{TM}}-22 \mathrm{~F}-\mathrm{cAMP}$ plasmid. After $10 \mathrm{~min}$ at room temperature, a single electrical pulse at $270 \mathrm{~V}$ and a capacitance of $950 \mu \mathrm{F}$ was delivered. The cell suspension was transferred to pre-warmed culture media and incubated for $24 \mathrm{~h}$ at $37^{\circ} \mathrm{C}$ in a humidified atmosphere of $5 \% \mathrm{CO}_{2}$.

\subsection{GloSensor assay}

The day before the experiment, cells expressing the biosensor were seeded into a 96-well clearbottomed white microplate in culture medium $(100 \mu \mathrm{L}$ per well $)$ and incubated at $37^{\circ} \mathrm{C}, 5 \% \mathrm{CO}_{2}$ overnight. The culture medium was then replaced with $\mathrm{CO}_{2}$-independent medium containing a $2 \%$ $\mathrm{v} / \mathrm{v}$ GloSensor cAMP reagent stock solution and $10 \% \mathrm{FBS}$. After $90 \mathrm{~min}$ of incubation at $37^{\circ} \mathrm{C}$, the luminescence was measured using a TriStar plate reader (Berthold Technologies; Thoiry, France; $100 \mathrm{~ms}$ integration). The luminescence was recorded before and after injection of the drugs (FSK, CHK, and vehicle).

\section{RESULTS}

\section{1 cAMP-inhibitory response to CHK is independent of the method of cAMP pre- stimulation (forskolin, inhibitor of phosphodiesterase, Gs-linked ligands)}

To explore the CHK-induced cAMP-inhibitory response, we stably expressed the luminescent biosensor GloSensor ${ }^{\mathrm{TM}}-22 \mathrm{~F}$ in a HEK293 cell clone that constitutively expressed CXCR4 and ectopically expressed CX3CR1 [18]. The HEK biosensor-expressing subclone - hereafter called HEKgloCX3CR1 - exhibited a stimulatory response to FSK, a classic adenylate cyclase (AC) activator [19] (Figure 1A, black trace). As expected, the FSK response was absent when using the parental HEK293 clone that does not express the biosensor (Figure 1A, dotted trace). To examine the cAMP-inhibitory responses, the CHK was added at the peak of the FSK signal (Figure 1A, thin arrow). Using $100 \mathrm{nM}$ of either CX3CL1 (Figure 1A, red traces) or CXCL12 (Figure 1A, green traces), we observed a significant inhibition of the signal, while the addition of the control CHK CCL2 left the cAMP response unchanged (Figure 1A, blue trace). The net response after addition of either of the three CHKs - i.e. difference between signals obtained in the presence (Figure 1A, blue, red and green traces) and absence of CHKs (Figure 1A, black trace) - is reported in Figure 1B (left side ordinate). Both CX3CL1 and CXCL12 signals reached a maximum peak 8-12 min after CHK addition and displayed a slow decay, with $50 \%$ of the peak amplitude after $40-50 \mathrm{~min}$. When expressed as a percentage of the maximum FSK control response (Figure 1B, right side ordinate), the amplitudes of the signals from both CX3CL1 and CXCL12 treatments were approximately 35$40 \%$ (Table 1). While the native, soluble form of CX3CL1 is not only composed of the CHK domain, as with other CHKs like CXCL12, but also a mucin stalk [20], we checked whether the full-length, soluble CX3CL1 evoked a response similar to the CX3CL1 CHK domain (Figure S1).

We next examined whether the response was conserved if $\mathrm{CHK}$ was added at a shorter time after FSK. We observed that the CX3CL1 response had a similar amplitude and shape when the CHK was added either 10 (Figures 1C, blue trace; Table 1) or 20 minutes (Table 1) after FSK, instead of 30 minutes. Beyond 30 minutes of FSK stimulation, the cAMP-related signal was observed to significantly decline (Figures 1A and S2A). This was surprising since a long-term weakening of the FSK action was never reported. However, such decay has already been observed in long-term FSK experiments, using the GloSensor [21, 22]. We noticed that this decay globally followed a hyperbolic curve (i.e. linear when plotted as $\log / \log$, Figure S2B), as is often observed in 
luciferase bioluminescence experiments $[23,24]$. So, this hyperbolic decline is probably due to intrinsic properties of the luminescence physical process. Whatever the origin of this long term decay, it was interesting to test the CHK response during its course. So we also added CX3CL1 60 min after FSK and observed a net decrease of the signal (Figure 1C, green trace). Expressing the CX3CL1 response in this case as a percentage of the control maximum as for the preceding experiments clearly gave a lower amplitude than for preceding responses. By contrast, when we expressed the results as a percentage of the luminescence gained when the CHK was added, the response was similar to the previous ones (Figure 1C and Table 1).

The CHK response was further analyzed as a function of the concentrations of FSK added before CHK treatment. The addition of $0.5,1,5$, and $20 \mu \mathrm{M}$ FSK produced signals with amplitudes from 300-4000 luminescence units (Figure S2A, thin traces). In each case, CX3CL1 induced a net inhibitory response (Figure S2A, bold traces). When expressed as a percentage of the maximum control signal, the responses to CX3CL1 were similar when using FSK concentrations of 0.5 or 1 $\mu \mathrm{M}$ (Figure 1D, black and red traces), with a peak in amplitude at approximately $30 \%$. With higher FSK concentrations, i.e., 5 and $20 \mu \mathrm{M}$ (Figure S2A, blue and green traces), the FSK signal was greater than 2000 luminescence units, and the amplitude of the subsequent CHK response was smaller (5-15\%) (Figure 1D, blue and green traces). The distortion observed at the highest FSK concentration was probably due to saturation of the biosensor, as indicated by the GloSensor manufacturer when luminescence exceeds 2000 units and confirmed when we used a permeant, non-hydrolysable cAMP analog (Figure S3). Taken together, our data indicate that the CHK response was robust and did not depend on the concentration of the pre-stimulatory compound or the time point of treatment during pre-stimulation, as long as the signal was kept in the linear range of biosensor activity.

Next, the effect of various CHK doses was analyzed. With all of the concentrations, we observed CX3CL1 responses with similar outlines (Figure 2A). The dose-response curves had similar EC50s, almost $0.1 \mathrm{nM}$, with two different FSK concentrations $(0.25$ and $1 \mu \mathrm{M})$ (Figure $2 \mathrm{~B})$. Moreover, the CX3CL1 responses were suppressed after pertussis toxin treatment (Figure 2A, grey trace), confirming that the CHK act through the Gi-pathway. Similar results were found with CXCL12 responses (data not shown).

According to previous studies of Gs-mediated cAMP-stimulatory responses [13, 25], phosphodiesterases (PDEs) are involved in both limiting the amplitude of cAMP responses and promoting their rapid decline. As expected, the addition of IBMX, a non-selective PDE inhibitor, considerably increased the FSK-induced cAMP signal (Figure S4A, compare red and black thin traces). By contrast, the amplitude of the CX3CL1 response was only slightly decreased as compared to that observed in the absence of IBMX (Figure S4B, red and black traces; Table 1). However, the response kinetics was noticeably slower: the maximum response appeared after more than 25 min (Table 1). We also analyzed the response to CX3CL1 treatment after addition of IBMX alone (Figure S4A, blue traces): we detected responses similar to that observed in the presence of FSK plus IBMX (Figure S4B, blue trace, Table 1).

We also tested a more physiological cAMP pre-stimulation, i.e., agonists of the GPCR coupled to Gs, such as the prostaglandin or $\beta_{2}$-adrenergic receptors present in HEK293 cells [26-28]. Addition of PGE2 to HEKgloCX3CR1 produced a signal similar to that evoked by FSK (Figure S5A, black trace) while the addition of the $\beta_{2}$-adrenergic agonist isoproterenol generated a signal gaining its maximum in less than 10 minutes and showing biphasic decay (Figure S5C black trace). The subsequent $\mathrm{CHK}$ responses (Figures S5B, S5D) were found to be similar to that observed when FSK was the prestimulatory compound, with comparable amplitude and time to maximum (Table 1). Finally, we checked the CHK responses were similar in other cell types, such as Jurkat and HPB-ALL (data not shown). Globally our data indicated that the CHK response was relatively independent of the cellular context and the prestimulatory conditions (nature and concentration of the stimulating agent, delay with which $\mathrm{CHK}$ is added). 


\subsection{Absence of cross desensitization in different CHK responses}

Because different CHKs seem to share the same signaling machinery, we wanted to test whether the addition of one CHK might suppress the subsequent response from another. After a full response to CX3CL1, a second treatment of CX3CL1 gave no signal at all (Figure 3A, trace a, bold arrows), while a second treatment of CXCL12 gave a clear response (Figure 3A, trace b, empty arrow). A similar pattern was observed in the reverse scenario, i.e., when CX3CL1 treatment followed CXCL12 (Figure 3A, trace c). Moreover, when the individual CX3CL1 responses were expressed as a percentage of the control at the time of CHK treatment, the signals were similar, regardless of whether the CX3CL1 was added after CXCL12 (Figure 3B, green trace corresponding to trace c), or after the control CHK CCL2 (Figure 3B, yellow trace corresponding to trace d). The same pattern was also found for CXCL12 responses (Figure 3C corresponding to traces $b$ and e). This indicates that (i) there was no cross desensitization between responses to two different CHKs and (ii) the intracellular machinery was not a limiting factor for the response amplitude of individual CHKs. To confirm this latter result, we simultaneously treated cells with CX3CL1 and CXCL12 (Figure 3A, trace $\mathrm{f}$ ). As expected, this produced a greater signal than a single CHK (more than 50\%); however, its amplitude did not appear to correspond to the mere addition of both single responses.

\subsection{Role of receptor internalization in the dynamics of the CHK response}

Recently, cellular cAMP responses have been shown to be elicited even when the receptors were internalized in both Gs-mediated responses [29-35] and Gi-mediated ones [36]. Therefore, we investigated the role of the surface CHK receptors in shaping the cAMP-inhibitory responses. To this end, we used the human CX3CR1 antagonist F1 that we recently identified using intracellular calcium signaling [37]. As expected, F1 was also a competitive antagonist for CX3CL1-evoked cAMP-inhibitory responses (Figure S6A): the double-reciprocal plot gave the same intercept in the presence and absence of the inhibitor, a hallmark of competitive inhibition (Figure S6B). Addition of a saturating concentration of F1 and CHK effectively suppressed the cAMP response (Figure 4A, compare traces a and $\mathrm{b}$ ). When added 8 or $15 \mathrm{~min}$ after CX3CL1, the antagonist elicited a rapid return to zero of the signal and shortened the length of the response (Figure 4A, traces $\mathrm{c}$ and $\mathrm{d}$ ). By contrast, F1 treatment after the response peak - at 30 or $60 \mathrm{~min}$ - had no effect (Figure 4A, traces e and $\mathrm{f}$ ), indicating that the surface active receptors were not involved in continuing the response. Consistent with this hypothesis, we found that the surface CX3CR1 receptor number decreased 30 min after CX3CL1 treatment and remained stable for 90 min (Figure 4B, black circles). Next, we used dynasore, an inhibitor of dynamin-mediated receptor internalization [38, 39]. After treatment with this compound, CX3CR1 internalization was completely abolished (Figure 4B, blue circles). This treatment slightly delayed the onset of the cAMP-inhibitory response, and its decay was significantly slowed down (Figure 4C, compare blue and black traces).

\section{DISCUSSION}

This study demonstrates that the cAMP biosensor is a valuable tool to delineate CHK-induced cAMP-inhibitory responses, particularly downstream of CX3CR1 and CXCR4. Biosensors based on luminescence [40] or FRET techniques [41-45] have been largely used to decipher the temporal dynamics of Gs- [28, 46, 47] and Gq-mediated [48, 49] cAMP-related activation. However, Gimediated cAMP-inhibitory signals are rarely monitored with these techniques [50]. While some studies have used the $22 \mathrm{~F}-G l o S e n$ sor $^{\mathrm{TM}}$ cAMP-luminescent biosensor to quantify Gi-mediated responses in HEK293 cells, they only used an end-point assay [21, 40]. In this study, we used this biosensor to monitor its kinetics. We primarily found that the responses evoked by CHKs, when expressed as a percentage of cAMP inhibition, were remarkably conserved regarding both their amplitude and kinetics. The CHK response seems limited to $30-40 \%$ inhibition, regardless of the manner of cAMP pre-stimulation and time of CHK treatment (Figure 1B, S5B and S5D; Table 1). The amplitude is of the same order of magnitude as the amplitude of CHK Gi-mediated responses found with conventional cellular cAMP assays $[4,5,51]$. In contrast to most methods that monitor 
cAMP-inhibitory response, the biosensor technique used here does not require a high concentration of FSK and simultaneous addition of IBMX. This allows measurements to be done in a more physiological context, i.e., in the presence of agonists of native inflammatory Gs-associated receptors (Figure S5). We noticed that the stimulatory response evoked by isoproterenol has a biphasic kinetics (Figure S5C) that is not observed with other biosensors in the same cell type [28]. This could be due to receptors differentially distributed in rafts [14, 52]. Anyway, the kinetics of the superimposed Gi-mediated chemokine response remains independent of these various stimulatory signals (Table 1$)$.

As expected, the main limiting factor in our observation of CHK-induced cAMP-inhibitory responses was the sensitivity and linear dynamic range of the biosensor: the height of the prestimulation signal had to be small enough to remain in the linear range of the biosensor and avoid saturation $(\leq 1 \mu \mathrm{M}$ FSK, Figure 1D). These limits globally match the recently described dynamic range of the 22F-GloSensor ${ }^{\mathrm{TM}}[22]$ and correspond to a cAMP concentration range of $10^{-9}-5.10^{-7} \mathrm{M}$, as illustrated in Figure S3 (insert).

Another characteristic of this method using GloSensor is the long-term decay of the stimulatory signals observed especially beyond 30 minutes after FSK. This decline could be caused by a loss of cellular ATP, the cofactor of luciferase, after extended cAMP synthesis or/and by a thermal aggregation of the firefly luciferase [24]. More probably it is due to intrinsic properties of the luminescence physical process, since this decay was surprisingly following a hyperbolic curve (i.e., linear when plotted as Log/Log, Figure S2B) [23, 24]. Anyway, this observed decay does not impair the real-time monitoring of the cAMP-inhibitory response, since the chemokine responses are similar even if chemokine is added 60 minutes after FSK (Figure 1C, Table 1).

\subsection{Parameters governing the kinetics of the CHK-induced cAMP-inhibitory response}

In a cellular context, the activity level of a biosensor, such as the cAMP-related GloSensor used in this study, is controlled by several parameters. One parameter is the apparent affinity of the biosensor binding domain for the cAMP molecule. It is relatively easy to elucidate the on-rate of a cAMP biosensor using permeant and non-hydrolyzable cAMP analogues (Figure S3). However, the rate-limiting step of a cAMP-inhibitory response is the off-rate of the cAMP from the biosensor site, and this reaction is harder to test. We found here that the Gi-mediated CHK response peaked after 8-12 min of CHK stimulation; so it is possible that cAMP release from the GloSensor could limit to some extent the onset of the cAMP-inhibitory responses. Moreover, a slow off-rate could account for the fact that the CHK-triggered cellular responses we observed often last $30 \mathrm{~min}$ or more. However, such long-time duration was also shown with other Gi-evoked second messengers, for example during extracellular signal-regulated kinase phosphorylation [53].

Another parameter involved in the dynamic response of the GloSensor is the turnover of the cellular cAMP produced by the ACs and degraded by the PDEs. It is known that the quicker the turnover, the quicker the response of the GloSensor to a drop in the cellular cAMP concentration. This could partly explain why the CHK response in our experiments was slower during both the onset and decline of the cAMP-inhibitory response in the presence of the PDE inhibitor IBMX (Figure S4 and Table 1; see also text below and Figure 6D).

Compartmentation of cellular cAMP is also a major parameter that governs the shape of the responses monitored using biosensors, as previously shown for cAMP-stimulating responses [54]. Indeed, diverse response kinetics have been observed according to the cellular localization of the biosensor, whether it was soluble or membrane-tethered [9], or associated in raft or non-raft microdomains [54]. The GloSensor ${ }^{\mathrm{TM}} 22 \mathrm{~F}$ used in this study is derived from the PKA-cAMP binding site [22] and is presumably soluble; so it responds to cAMP changes throughout the cytosolic compartment of the cell. If CHK-induced cAMP-inhibitory responses are confined to a subcellular compartment through the co-localization of receptors, Gi-proteins, ACs, and PDEs into a "receptosome", which has been shown for cAMP-stimulatory responses, the signals we observed 
represent the integration of many local responses, with delayed kinetics and average amplitudes. Use of a membrane-associated biosensor might provide access to such subcellular details [54].

Regardless of the localization of the biosensor used in this study, we showed that the PDEs had a role in shaping the observed signal. In the presence of the IBMX inhibitor, the FSK signal was increased ten-fold (Figure S4A, compare red and black traces), and the resting signal increased more than 100-fold (Figure S4A, blue trace), indicating that the cellular cAMP concentration was a result of basal AC activity vigorously counterbalanced by high PDE activity. One could hypothesize that, similar to Gs-mediated responses whose decay is associated with the activation of PDE [13, 25], the decay of Gi-mediated CHK responses could be associated with a large inhibition of PDE; however, this is not consistent with our data: the CHK response was only marginally affected by the presence of IBMX (Figure S4B; Table 1). This indicates that the response decay does not rely on inhibition of IBMX-sensitive PDE activity associated with Gi-mediated AC inhibition. Alternatively, one could suppose that PDEs insensitive to IBMX could exist in HEK293 cells, or that cells could reduce their cellular cAMP in another other way that has yet to be described. Anyway, our data confirmed that the cAMP-inhibitory response evoked by CHK is due to the Gimediated inhibition of the AC activity, either at is basal level (Figure S4, blue traces) or at is level stimulated by FSK (Figure S4, red and black traces).

The main result from this study is the finding that the amplitude of the CHK response is conserved and approximately $35-40 \%$, regardless of the method used to pre-stimulate the cAMP response. By comparison, a recent study found that responses downstream of metabotropic glutamate receptors assayed with the same biosensor in $\mathrm{CHO}$ cells reached an amplitude of $80 \%$ [50]. In our study, we showed that the intracellular signaling machinery was not a limiting factor for the response because simultaneous treatment with two different $\mathrm{CHKs}$ produced a greater response than individual treatments (Figure 4A, trace f). Moreover, sequential treatments with two different CHKs gave two individual responses (Figure 4A, traces $\mathrm{b}$ and $\mathrm{c}$ ), while sequential treatments with the same CHK resulted in only one response: the receptors became saturated by the first treatment and were unavailable to transduce further signals with the second treatment (Figure 4A, trace a). This behavior was reminiscent of the CHK calcium responses [55] and indicates that the number of available surface $\mathrm{CHK}$ receptors likely limits the $\mathrm{CHK}$ response to $40 \%$. This was further demonstrated with the use of the competitive CX3CL1 antagonist: the amplitude of the response followed the rate of receptor occupancy of the CX3CL1 agonist (Figure S6). Moreover, when the antagonist concentration was sufficient to displace CX3CL1 during the onset of the response, there was a rapid return to the basal signal level (Figure 4A), indicating that the number of liganded receptors directly governs the response profile.

The use of biosensors has provoked an important paradigm shift in GPCR signaling, demonstrating that some receptor-induced Gs-mediated cAMP production persists after endosome-induced receptor internalization [29-32, 34, 35]. This has also been shown for a Gi-dependent signals downstream of the sphingosine-1-phosphate receptor 1 [36]. Therefore, it was important to evaluate the potential existence of such signaling from internalized CHK receptors in our model, especially because it is controversial whether this non-classical persistent signaling exists in HEK293 cells $[56,57]$. We found that the onset of the CHK response followed the kinetics of internalization (Figure 4). Moreover, the putative persistent endosomal signaling would be suppressed under conditions where receptor internalization was inhibited [35]: this was clearly not the case here (Figure 4C). Therefore, we conclude that the CHK responses we observed are mainly driven by the availability of the cognate surface $\mathrm{CHK}$ receptors.

\subsection{Modeling the $\mathrm{CHK}$ response}

We attempted to model the global shape of the CHK response using two simple assumptions: (a) the cellular cAMP concentration at each time point is only a function of synthesis by AC and degradation by PDEs and (b) the degradation rate of the PDEs is a linear function of the cAMP concentration at each time point, as for enzymes working in a substrate concentration range equal or 
less to their $\mathrm{K}_{\mathrm{m}}$. Indeed the $\mathrm{K}_{\mathrm{m}}$ of PDE4, the main PDE subtype present in HEK293 cells [49, 58], is of $1 \mu \mathrm{M}$ or above [59], that is largely higher than the cellular cAMP concentration range here found (Figure S3). We then supposed that the primary cause of the observed signal is exclusively the variations of the AC activity (here denoted $\alpha$ ), i.e. either its activation by FSK (Figure 5A) or its inhibition by $\mathrm{CHK}$ (Figure 5B) giving the variations of the cellular cAMP concentration (here denoted $\mathrm{C}$ ) given in Figures 5C or 5D respectively, according to the following calculations.

So the above assumptions (a) and (b) give the following differential equation (1):

$\delta \mathrm{C} / \delta \mathrm{t}=\alpha-\mathrm{C}^{*} \beta=\alpha-\mathrm{C} / \tau_{\beta}$

where $\mathrm{C}$ is the cellular cAMP concentration at time $\mathrm{t}, \alpha$ the rate of cAMP synthesis by AC in M.min ${ }^{-1}, \beta$ the proportion of cAMP degradation by PDE in $\min ^{-1}$, and $\tau_{\beta}$ the characteristic time of this cAMP degradation by $\operatorname{PDE}\left(\tau_{\beta}=1 / \beta\right)$.

Postulating that the addition of FSK at time $t_{0}$ results in a rapid increase in the cellular AC activity from $\alpha_{\mathrm{i}}$ to $\alpha_{0}$ (Figure 5A), $\mathrm{C}$ is given by the following equation (2), as displayed in Figure 5C:

$\mathrm{C}(\mathrm{t})=\mathrm{C}_{0}+\left(\mathrm{C}_{\mathrm{f}}-\mathrm{C}_{0}\right)\left(1-\exp \left(-\left(\mathrm{t}-\mathrm{t}_{0}\right) / \tau_{\beta}\right)\right)$

where $\mathrm{C}_{0}$ is the initial cAMP concentration at time $\mathrm{t}_{0}$ and $\mathrm{C}_{\mathrm{f}}$ is the final cAMP concentration, here equal to $\alpha_{0} * \tau_{\beta}$.

We then postulated that the addition of a $\mathrm{CHK}$ at time $\mathrm{t}_{1}$ results in a rapid decrease in the cellular AC activity from $\alpha_{0}$ to $\mathrm{p}^{*} \alpha_{0}(0<\mathrm{p}<1)$ and that it returns to $\alpha_{1}$ exponentially with a characteristic time $\tau_{1}$. Then, $\alpha$ becomes $\alpha_{\mathrm{CHK}}$ and is given by the following equation (3), as displayed in Figure 5B:

$$
\alpha_{\mathrm{CHK}}(\mathrm{t})=\alpha_{0}-\alpha_{0}(1-\mathrm{p})\left(1-\exp \left(-\left(\mathrm{t}-\mathrm{t}_{1}\right) / \tau_{1}\right)\right)
$$

We can derive the cellular cAMP concentration $\mathrm{C}_{\mathrm{CHK}}$ for $\mathrm{t}>\mathrm{t}_{1}$. Assuming that $\mathrm{C}$ starts at a constant value $\mathrm{C}_{1}$ for $\mathrm{t} \leq \mathrm{t}_{1}$, we can derive the following equation (4), as displayed in Figure 5D:

$$
\mathrm{C}_{\mathrm{CHK}}(\mathrm{t})=\mathrm{C}_{1}-\mathrm{C}_{1}(1-\mathrm{p}) \tau_{1} /\left(\tau_{1}-\tau_{\beta}\right)\left(\exp \left(-\left(\mathrm{t}-\mathrm{t}_{1}\right) / \tau_{1}\right)-\exp \left(-\left(\mathrm{t}-\mathrm{t}_{1}\right) / \tau_{\beta}\right)\right)(4)
$$

Using equation (2), it is possible to fit the data from Figure 1A (black trace), i.e., the second half of the FSK signal onset (Figure $6 \mathrm{~A}$, red trace). Then, we can calculate the CHK response $\Delta \mathrm{C}_{\mathrm{CHK}}(\mathrm{t})$ given by equation (5):

$$
\begin{aligned}
& \Delta \mathrm{C}_{\mathrm{CHK}}(\mathrm{t})=\left(\mathrm{C}_{\mathrm{CHK}}(\mathrm{t})-\mathrm{C}(\mathrm{t})\right) /(\max \mathrm{C}(\mathrm{t}))=-(1-\mathrm{p}) \tau_{1} /\left(\tau_{1}-\tau_{\beta}\right)\left(\exp \left(-\left(\mathrm{t}-\mathrm{t}_{1}\right) / \tau_{1}\right)-\exp (-(\mathrm{t}-\right. \\
& \left.\left.\left.\mathrm{t}_{1}\right) / \tau_{\beta}\right)\right)(5)
\end{aligned}
$$

This simple equation that provides $\Delta \mathrm{C}_{\mathrm{CHK}}(\mathrm{t})$ is valid even in the general case where $\mathrm{C}(\mathrm{t})$ is not constant for $\mathrm{t} \leq \mathrm{t}_{1}$ (Supplementary Text S7). Using the $\tau_{\beta}$ value found by fitting the FSK signal (Figure 6A, red trace), we were able to calculate the CHK response, e.g. the response obtained when the CHK was added 30 min after FSK stimulation, i.e., at the maximum of the FSK signal. Setting $\mathrm{t}_{1}=\mathrm{t}_{0}+30$, our model (Figure $6 \mathrm{~B}$, red trace) fits well with the onset of the observed response (Figure 5B, black trace) and the first half of its decay . We noticed that equation (5), which gives $\Delta \mathrm{C}_{\mathrm{CHK}}(\mathrm{t})$, does not depend on $\mathrm{t}_{0}$, i.e., the time point of FSK treatment; it only depends on $(\mathrm{t}-$ $\mathrm{t}_{1}$ ). This means that, according to our model, the outline of the CHK response does not depend on the delay between CHK and FSK treatments. This is exactly what we observed with our experimental data (Figure 1C).

However, our present fit (hereafter called model A) did not account for the decay of the FSK signal observed after 20-30 min. This hyperbolic decay (Figure S2B) was taken into account (Supplementary text S7) giving a refined model called model B. This model provides a better fit, not only for the FSK signal (Figure 6A, blue trace), but also for the CHK response (Figure 6B, blue trace), especially for the second part of the response decline.

Since both models gave similar results for most of the CHK response kinetics, we used model A for further calculations because it is simpler. Using equation (4), we also fit the responses to different CX3CL1 doses (Figure 2A) by changing the $\mathrm{p}$ factor as expected (Figure 6C); its value is 0.65, 
$0.74,0.79$, and 0.85 for the CX3CL1 concentrations of $5,2.5,1$, and $0.3 \mathrm{nM}$, respectively. We also fit the CHK response in the presence of IBMX (Figure S4, blue trace) by decreasing the $\beta$ parameter representing the PDE activity: in this case (Figure 6D), $\beta$ is more than 2 -fold decreased, increasing $\tau_{\beta}$ from 4.7 (Figure $6 \mathrm{D}$, red trace) to $10.8 \mathrm{~min}$ (Figure $6 \mathrm{D}$, orange trace). Finally, we used our model to fit the response observed in the presence of dynasore (Figure $4 \mathrm{C}$, blue trace) by increasing the $\tau_{1}$ parameter, which represents the characteristic time of the return of $\alpha$ to its initial value. Its value increases from 46 (Figure 6E, red trace) to $250 \mathrm{~min}$ (Figure 6E, orange trace). Taken together, we can conclude that the kinetics of the $\mathrm{CHK}$ responses observed with the luminescent biosensor can be reproduced with a simple model of cellular cAMP production and hydrolysis.

\subsection{Conclusion}

As mentioned in the introduction, the contribution of the cAMP second messenger in the functional role of CHKs remains controversial. While it does not seem to be directly involved in chemotaxis [7], cAMP could either positively [6] or negatively [5, 60] influence the CHK-induced chemotactic activity of monocytes. Moreover, the cAMP-EPAC (exchange protein directly activated by cAMP) pathway seems to contribute to the synthesis of CHKs [61]. The adhesion of monocytes to HUVEC is largely decreased in the presence of cAMP $[62,63]$ through a mechanism involving PKA and phosphorylated RhoA [64]. By contrast, the cAMP-EPAC pathway stimulates monocyte adhesion in activated HUVEC [6]. Finally, cAMP has been shown to be an important mediator of growth arrest and apoptosis in B and T cells, while it delays apoptosis in neutrophils, likely through EPAC activation [65]. Further work is required to elucidate the functional correlates in the CHK-induced cAMP-inhibitory responses. Nevertheless, the remarkable conservation of the response signal, regardless of the mode of cAMP pre-stimulation, indicates the robustness of the CHK effects, which do not depend on the preceding cellular cAMP level, meaning that each CHK exert their influence in all inflammatory contexts, in a manner independent of others CHKs.

\section{ACKNOWLEDGEMENTS}

We would like to thank Clotilde Randriamampita (Institut Cochin, CNRS UMR8104 INSERM U567, Paris), Jérôme Delon (Institut Cochin, CNRS UMR8104 INSERM U567, Paris), and Pierre Vincent (Neurobiologie des Processus Adaptatifs, CNRS, UPMC, UMR7102) for their helpful discussions. This work was supported by grants from the "Agence Nationale de la Recherche" (Adhekine $n^{\circ}$ 09-PIRI-0003-01, I-ckaire n॰12-EMMA-0050) and European Community's framework programme FP7/2007-2013 (ENDOSTEM n 241440) and from INSERM, UPMC, and CNRS.

\section{AUTHORS' CONTRIBUTIONS}

Virginia Felouzis performed all of the experiments. Patricia Hermand and Philippe Deterre planned the experiments and helped with the data analysis. Guy Trambly developed the model to fit the data. Virginia Felouzis, Guy Trambly, Christophe Combadière, and Philippe Deterre wrote the paper. 


\section{BIBLIOGRAPHY}

[1] M. Baggiolini, Chemokines and leucocyte traffic, Nature, 392 (1998) 565-568.

[2] A. Viola, A.D. Luster, Chemokines and their receptors: drug targets in immunity and inflammation, Annu Rev Pharmacol Toxicol, 48 (2008) 171-197.

[3] G. Shi, S. Partida-Sanchez, R.S. Misra, M. Tighe, M.T. Borchers, J.J. Lee, M.I. Simon, F.E. Lund, Identification of an alternative $\mathrm{G}\{$ alpha\}q-dependent chemokine receptor signal transduction pathway in dendritic cells and granulocytes, J. Exp. Med., 204 (2007) 2705-2718.

[4] S.J. Myers, L.M. Wong, I.F. Charo, Signal Transduction and Ligand Specificity of the Human Monocyte Chemoattractant Protein-1 Receptor in Transfected Embryonic Kidney Cells, Journal of Biological Chemistry, 270 (1995) 5786-5792.

[5] G. O'Boyle, J.G. Brain, J.A. Kirby, S. Ali, Chemokine-mediated inflammation: Identification of a possible regulatory role for CCR2, Mol Immunol, 44 (2007) 1944-1953.

[6] M.J. Lorenowicz, J. van Gils, M. de Boer, P.L. Hordijk, M. Fernandez-Borja, Epac1-Rap1 signaling regulates monocyte adhesion and chemotaxis, J Leukoc Biol, 80 (2006) 1542-1552.

[7] K. Jarnagin, D. Grunberger, M. Mulkins, B. Wong, S. Hemmerich, C. Paavola, A. Bloom, S. Bhakta, F. Diehl, R. Freedman, D. McCarley, I. Polsky, A. Ping-Tsou, A. Kosaka, T.M. Handel, Identification of surface residues of the monocyte chemotactic protein 1 that affect signaling through the receptor CCR2, Biochemistry, 38 (1999) 16167-16177.

[8] T.C. Rich, J.W. Karpen, Review article: cyclic AMP sensors in living cells: what signals can they actually measure?, Ann Biomed Eng, 30 (2002) 1088-1099.

[9] D. Willoughby, D.M. Cooper, Live-cell imaging of cAMP dynamics, Nature methods, 5 (2008) 29-36.

[10] J.P. Vilardaga, M. Bunemann, T.N. Feinstein, N. Lambert, V.O. Nikolaev, S. Engelhardt, M.J. Lohse, C. Hoffmann, GPCR and G proteins: drug efficacy and activation in live cells, Molecular endocrinology, 23 (2009) 590-599.

[11] L.R. Castro, E. Guiot, M. Polito, D. Paupardin-Tritsch, P. Vincent, Decoding spatial and temporal features of neuronal cAMP/PKA signaling with FRET biosensors, Biotechnol J, 9 (2014) 192-202.

[12] M.J. Lohse, S. Nuber, C. Hoffmann, Fluorescence/bioluminescence resonance energy transfer techniques to study G-protein-coupled receptor activation and signaling, Pharmacological Reviews, 64 (2012) 299-336.

[13] T.C. Rich, K.A. Fagan, T.E. Tse, J. Schaack, D.M. Cooper, J.W. Karpen, A uniform extracellular stimulus triggers distinct cAMP signals in different compartments of a simple cell, Proc Natl Acad Sci U S A, 98 (2001) 13049-13054.

[14] L.M. DiPilato, X. Cheng, J. Zhang, Fluorescent indicators of cAMP and Epac activation reveal differential dynamics of cAMP signaling within discrete subcellular compartments, Proc Natl Acad Sci U S A, 101 (2004) 16513-16518.

[15] M. Mongillo, T. McSorley, S. Evellin, A. Sood, V. Lissandron, A. Terrin, E. Huston, A. Hannawacker, M.J. Lohse, T. Pozzan, M.D. Houslay, M. Zaccolo, Fluorescence resonance energy transfer-based analysis of cAMP dynamics in live neonatal rat cardiac myocytes reveals distinct functions of compartmentalized phosphodiesterases, Circulation research, 95 (2004) 67-75.

[16] L.R. Castro, M. Brito, E. Guiot, M. Polito, C.W. Korn, D. Herve, J.A. Girault, D. PaupardinTritsch, P. Vincent, Striatal neurones have a specific ability to respond to phasic dopamine release, J Physiol, 591 (2013) 3197-3214.

[17] M. Daoudi, Etude fonctionnelle des variants naturels du CX3CR1, récepteur de la chimiokine Fractalkine. Adhérence et transduction du signal., Thèse Doctorat Paris XI, (2005).

[18] M. Daoudi, E. Lavergne, A. Garin, N. Tarantino, P. Debre, F. Pincet, C. Combadiere, P. Deterre, Enhanced Adhesive Capacities of the Naturally Occurring Ile249-Met280 Variant of the Chemokine Receptor CX3CR1, J. Biol. Chem., 279 (2004) 19649-19657.

[19] K.B. Seamon, W. Padgett, J.W. Daly, Forskolin: unique diterpene activator of adenylate 
cyclase in membranes and in intact cells, Proc Natl Acad Sci U S A, 78 (1981) 3363-3367.

[20] J.F. Bazan, K.B. Bacon, G. Hardiman, W. Wang, K. Soo, D. Rossi, D.R. Greaves, A. Zlotnik, T.J. Schall, A new class of membrane-bound chemokine with a CX3C motif, Nature, 385 (1997) 640-644.

[21] C.T. Gilliland, C.L. Salanga, T. Kawamura, J. Trejo, T.M. Handel, The Chemokine Receptor CCR1 Is Constitutively Active, Which Leads to G Protein-independent, \{beta\}-Arrestinmediated Internalization, J. Biol. Chem., 288 (2013) 32194-32210.

[22] B.F. Binkowski, B.L. Butler, P.F. Stecha, C.T. Eggers, P. Otto, K. Zimmerman, G. Vidugiris, M.G. Wood, L.P. Encell, F. Fan, K.V. Wood, A luminescent biosensor with increased dynamic range for intracellular cAMP, ACS chemical biology, 6 (2011) 1193-1197.

[23] D. Ke, S.-C. Tu, Activities, Kinetics and Emission Spectra of Bacterial Luciferase-Fluorescent Protein Fusion Enzymes, Photochemistry and Photobiology, 87 (2011) 1346-1353.

[24] S. Rasouli, S. Hosseinkhani, P. Yaghmaei, A. Ebrahim-Habibi, Effects of Sucrose and Trehalose on Stability, Kinetic Properties, and Thermal Aggregation of Firefly Luciferase, Appl Biochem Biotechnol, 165 (2011) 572-582.

[25] T.C. Rich, T.E. Tse, J.G. Rohan, J. Schaack, J.W. Karpen, In vivo assessment of local phosphodiesterase activity using tailored cyclic nucleotide-gated channels as cAMP sensors, J Gen Physiol, 118 (2001) 63-78.

[26] S. Desai, B. Ashby, Agonist-induced internalization and mitogen-activated protein kinase activation of the human prostaglandin EP4 receptor, FEBS Letters, 501 (2001) 156-160.

[27] R. Morath, T. Klein, H.W. Seyberth, R.M. Nusing, Immunolocalization of the four prostaglandin E2 receptor proteins EP1, EP2, EP3, and EP4 in human kidney, Journal of the American Society of Nephrology: JASN, 10 (1999) 1851-1860.

[28] J.D. Violin, L.M. DiPilato, N. Yildirim, T.C. Elston, J. Zhang, R.J. Lefkowitz, beta2adrenergic receptor signaling and desensitization elucidated by quantitative modeling of real time cAMP dynamics, The Journal of biological chemistry, 283 (2008) 2949-2961.

[29] S. Ferrandon, T.N. Feinstein, M. Castro, B. Wang, R. Bouley, J.T. Potts, T.J. Gardella, J.P. Vilardaga, Sustained cyclic AMP production by parathyroid hormone receptor endocytosis, Nature Chem Biology, 5 (2009) 734-742.

[30] D. Calebiro, V.O. Nikolaev, M.C. Gagliani, T. de Filippis, C. Dees, C. Tacchetti, L. Persani, M.J. Lohse, Persistent cAMP-signals triggered by internalized G-protein-coupled receptors, PLoS Biol, 7 (2009) e1000172.

[31] R.S. Kuna, S.B. Girada, S. Asalla, J. Vallentyne, S. Maddika, J.T. Patterson, D.L. Smiley, R.D. DiMarchi, P. Mitra, Glucagon-like peptide-1 receptor-mediated endosomal cAMP generation promotes glucose-stimulated insulin secretion in pancreatic beta-cells, American journal of physiology. Endocrinology and metabolism, 305 (2013) E161-170.

[32] V.L. Wehbi, H.P. Stevenson, T.N. Feinstein, G. Calero, G. Romero, J.-P. Vilardaga, Noncanonical GPCR signaling arising from a PTH receptor-arrestin-G\{beta $\{$ gamma $\}$ complex, PNAS, 110 (2013) 1530-1535.

[33] R. Irannejad, J.C. Tomshine, J.R. Tomshine, M. Chevalier, J.P. Mahoney, J. Steyaert, S.G.F. Rasmussen, R.K. Sunahara, H. El-Samad, B. Huang, M. von Zastrow, Conformational biosensors reveal GPCR signalling from endosomes, Nature, 495 (2013) 534-538.

[34] R. Irannejad, M. von Zastrow, GPCR signaling along the endocytic pathway, Curr Opin Cell Biol, 27 (2014) 109-116.

[35] J.P. Vilardaga, F.G. Jean-Alphonse, T.J. Gardella, Endosomal generation of cAMP in GPCR signaling, Nature chemical biology, 10 (2014) 700-706.

[36] F. Mullershausen, F. Zecri, C. Cetin, A. Billich, D. Guerini, K. Seuwen, Persistent signaling induced by FTY720-phosphate is mediated by internalized S1P1 receptors, Nat Chem Biol, 5 (2009) 428-434.

[37] K. Dorgham, A. Ghadiri, P. Hermand, M. Rodero, L. Poupel, M. Iga, O. Hartley, G. Gorochov, C. Combadiere, P. Deterre, An engineered CX3CR1 antagonist endowed with antiinflammatory activity, J Leukoc Biol, 86 (2009) 903-911. 
[38] T. Kirchhausen, E. Macia, H.E. Pelish, Use of Dynasore, the Small Molecule Inhibitor of Dynamin, in the Regulation of Endocytosis, in: C.J.D. William E. Balch, H. Alan (Eds.) Methods in Enzymology, Academic Press2008, pp. 77-93.

[39] Y.W. Huang, P. Su, G.Y. Liu, M.R. Crow, D. Chaukos, H. Yan, L.A. Robinson, Constitutive endocytosis of the chemokine CX3CL1 prevents its degradation by cell surface metalloproteases, J Biol Chem, 284 (2009) 29644-29653.

[40] K.N. Nobles, K. Xiao, S. Ahn, A.K. Shukla, C.M. Lam, S. Rajagopal, R.T. Strachan, T.Y. Huang, E.A. Bressler, M.R. Hara, S.K. Shenoy, S.P. Gygi, R.J. Lefkowitz, Distinct phosphorylation sites on the beta(2)-adrenergic receptor establish a barcode that encodes differential functions of beta-arrestin, Science signaling, 4 (2011) ra51.

[41] B. Ponsioen, J. Zhao, J. Riedl, F. Zwartkruis, G. van der Krogt, M. Zaccolo, W.H. Moolenaar, J.L. Bos, K. Jalink, Detecting cAMP-induced Epac activation by fluorescence resonance energy transfer: Epac as a novel cAMP indicator, EMBO Rep, 5 (2004) 1176-1180.

[42] G.N. van der Krogt, J. Ogink, B. Ponsioen, K. Jalink, A comparison of donor-acceptor pairs for genetically encoded FRET sensors: application to the Epac cAMP sensor as an example, PLoS ONE, 3 (2008) e1916.

[43] V.O. Nikolaev, M. Bunemann, L. Hein, A. Hannawacker, M.J. Lohse, Novel single chain cAMP sensors for receptor-induced signal propagation, The Journal of biological chemistry, 279 (2004) 37215-37218.

[44] V.O. Nikolaev, M. Bunemann, E. Schmitteckert, M.J. Lohse, S. Engelhardt, Cyclic AMP imaging in adult cardiac myocytes reveals far-reaching betal-adrenergic but locally confined beta2-adrenergic receptor-mediated signaling, Circulation research, 99 (2006) 1084-1091.

[45] C. Depry, M.D. Allen, J. Zhang, Visualization of PKA activity in plasma membrane microdomains, Mol Biosyst, 7 (2011) 52-58.

[46] J.B. Klarenbeek, J. Goedhart, M.A. Hink, T.W.J. Gadella, K. Jalink, A mTurquoise-Based cAMP Sensor for Both FLIM and Ratiometric Read-Out Has Improved Dynamic Range, PLoS ONE, 6 (2011) e19170.

[47] M.D. Allen, J. Zhang, Subcellular dynamics of protein kinase A activity visualized by FRETbased reporters, Biochem Biophys Res Commun, 348 (2006) 716-721.

[48] J.X. Shen, S. Wachten, M.L. Halls, K.L. Everett, D.M.F. Cooper, Muscarinic receptors stimulate AC2 by novel phosphorylation sites, whereas G $\beta \gamma$ subunits exert opposing effects depending on the G-protein source, Biochemical Journal, 447 (2012) 393-405.

[49] J.X. Shen, D.M.F. Cooper, AKAP79, PKC, PKA and PDE4 participate in a Gq-linked muscarinic receptor and adenylate cyclase 2 cAMP signalling complex, Biochemical Journal, 455 (2013) 47-56.

[50] J.O. DiRaddo, E.J. Miller, H.A. Hathaway, E. Grajkowska, B. Wroblewska, B.B. Wolfe, D.C. Liotta, J.T. Wroblewski, A real-time method for measuring cAMP production modulated by Galphai/o-coupled metabotropic glutamate receptors, The Journal of pharmacology and experimental therapeutics, 349 (2014) 373-382.

[51] V. Shyamala, H. Khoja, M. Moghadam, Inhibition of adenylyl cyclase by alpha chemokines IL-8 and GRO-alpha in Chinese hamster ovary cells expressing R1 and R2 receptors, Journal of interferon \& cytokine research : the official journal of the International Society for Interferon and Cytokine Research, 18 (1998) 235-239.

[52] L.M. DiPilato, J. Zhang, The role of membrane microdomains in shaping beta2-adrenergic receptor-mediated cAMP dynamics, Molecular bioSystems, 5 (2009) 832-837.

[53] B. Cambien, M. Pomeranz, H. Schmid-Antomarchi, M.A. Millet, V. Breittmayer, B. Rossi, A. Schmid-Alliana, Signal transduction pathways involved in soluble fractalkine-induced monocytic cell adhesion, Blood, 97 (2001) 2031-2037.

[54] S.R. Agarwal, P.C. Yang, M. Rice, C.A. Singer, V.O. Nikolaev, M.J. Lohse, C.E. Clancy, R.D. Harvey, Role of membrane microdomains in compartmentation of cAMP signaling, PLoS ONE, 9 (2014) e95835.

[55] C. Combadiere, S.K. Ahuja, J. Van Damme, H.L. Tiffany, J.L. Gao, P.M. Murphy, Monocyte 
chemoattractant protein-3 is a functional ligand for $\mathrm{CC}$ chemokine receptors 1 and $2 \mathrm{~B}$, J.Biol.Chem., 270 (1995) 29671-29675.

[56] R.C. Werthmann, S. Volpe, M.J. Lohse, D. Calebiro, Persistent cAMP signaling by internalized TSH receptors occurs in thyroid but not in HEK293 cells, FASEB journal : official publication of the Federation of American Societies for Experimental Biology, 26 (2012) 20432048.

[57] E. Geras-Raaka, S. Neumann, M.C. Gershengorn, Persistent cAMP signaling by TSH receptors revealed by phosphodiesterase inhibition, Thyroid, 23 (2013) 1484-1489.

[58] M.J. Lynch, G.S. Baillie, A. Mohamed, X. Li, C. Maisonneuve, E. Klussmann, G. van Heeke, M.D. Houslay, RNA Silencing Identifies PDE4D5 as the Functionally Relevant cAMP Phosphodiesterase Interacting with $\beta$ Arrestin to Control the Protein Kinase A/AKAP79mediated Switching of the $\beta 2$-Adrenergic Receptor to Activation of ERK in HEK293B2 Cells, Journal of Biological Chemistry, 280 (2005) 33178-33189.

[59] A.T. Bender, J.A. Beavo, Cyclic nucleotide phosphodiesterases: molecular regulation to clinical use, Pharmacological Reviews, 58 (2006) 488-520.

[60] J.S. Fine, H.D. Byrnes, P.J. Zavodny, R.W. Hipkin, Evaluation of signal transduction pathways in chemoattractant-induced human monocyte chemotaxis, Inflammation, 25 (2001) 61-67.

[61] A.L. Hertz, A.T. Bender, K.C. Smith, M. Gilchrist, P.S. Amieux, A. Aderem, J.A. Beavo, Elevated cyclic AMP and PDE4 inhibition induce chemokine expression in human monocytederived macrophages, Proceedings of the National Academy of Sciences, 106 (2009) 2197821983.

[62] A. Chigaev, A. Waller, O. Amit, L.A. Sklar, Galphas-coupled receptor signaling actively down-regulates alpha4beta1-integrin affinity: a possible mechanism for cell de-adhesion, BMC Immunol, 9 (2008) 26.

[63] D. Mori, H. Ishii, C. Kojima, N. Nitta, K. Nakajima, M. Yoshida, Cilostazol inhibits monocytic cell adhesion to vascular endothelium via upregulation of cAMP, J Atheroscler Thromb, 14 (2007) 213-218.

[64] C. Laudanna, J.J. Campbell, E.C. Butcher, Elevation of intracellular cAMP inhibits RhoA activation and integrin-dependent leukocyte adhesion induced by chemoattractants, J Biol Chem, 272 (1997) 24141-24144.

[65] M. Grandoch, V. Bujok, D. Fleckenstein, M. Schmidt, J.W. Fischer, A.A. Weber, Epac inhibits apoptosis of human leukocytes, Journal of Leukocyte Biology, 86 (2009) 847-849. 


\section{Table}

Table 1. Chemokine response characteristics according to the preactivation conditions

\begin{tabular}{|c|c|c|c|c|}
\hline $\begin{array}{l}\text { Preactivating } \\
\text { molecules }\end{array}$ & CHK & Delay & $\begin{array}{l}\text { Maximum of the } \\
\text { response (\% of } \\
\text { control max) } \\
\end{array}$ & $\begin{array}{c}\text { Time at } \\
\text { maximum } \\
(\min )\end{array}$ \\
\hline $0.5 \mu \mathrm{M}$ FSK & 50nM CX3CL1 & $30 \mathrm{~min}$ & $37.7 \pm 6.8$ & $11,9 \pm 1,7$ \\
\hline $0.5 \mu \mathrm{M}$ FSK & 50nM CXCL12 & $30 \mathrm{~min}$ & $36.6 \pm 9.6$ & $8.1 \pm 1,7$ \\
\hline $0.5 \mu \mathrm{M}$ FSK & 50nM CX3CL1 & $10 \mathrm{~min}$ & $39.8 \pm 5.5$ & $11.8 \pm 2.7$ \\
\hline $0.5 \mu \mathrm{M}$ FSK & 50nM CX3CL1 & $20 \mathrm{~min}$ & $40.4 \pm 4.4$ & $11.3 \pm 4.1$ \\
\hline $0.5 \mu \mathrm{M}$ FSK & 50nM CX3CL1 & $60 \mathrm{~min}$ & $35.9 \pm 11.2$ & $8.7 \pm 4.3$ \\
\hline $0.05 \mu \mathrm{M}$ PGE2 & 50nM CX3CL1 & $22 \mathrm{~min}$ & $36.4 \pm 3.2$ & $8.8 \pm 3.6$ \\
\hline $10 \mu \mathrm{M}$ ISO & 50nM CX3CL1 & $22 \mathrm{~min}$ & $30.3 \pm 4.6$ & $9.3 \pm 4.7$ \\
\hline $\begin{array}{c}0.03 \mu \mathrm{M} \text { FSK }+ \\
0.5 \mathrm{mM} \text { IBMX }\end{array}$ & 50nM CX3CL1 & $30 \mathrm{~min}$ & $30.1 \pm 0.9$ & $27.8 \pm 2.3^{* * *}$ \\
\hline $0.5 \mathrm{mM}$ IBMX & 50nM CX3CL1 & $30 \mathrm{~min}$ & $31.3 \pm 6.9$ & $30.0 \pm 4.8^{* * *}$ \\
\hline 50nM CXCL12 & 50nM CX3CL1 & $30 \mathrm{~min}$ & $32.8 \pm 8.7$ & $9.9 \pm 1.7$ \\
\hline 50nM CX3CL1 & 50nM CXCL12 & $30 \mathrm{~min}$ & $40.8 \pm 6.9$ & $9.4 \pm 0.9$ \\
\hline
\end{tabular}

Data of the two first lines are from four independent experiments and expressed as mean $\pm \mathrm{SD}$. The others are in quadruplicate and expressed as mean $\pm \mathrm{SD}$. The data are analyzed by 1-way ANOVA test of variance and Tukey post-analysis test. $* * *$ indicates $p<0.001$ as compared to the condition of the first line. 


\section{FIGURE LEGENDS}

Figure 1. The CAMP-inhibitory response triggered by CX3CL1 is independent of the method of cAMP pre-stimulation

(A) Luminescence signal obtained in the HEK293 parental cell line (black dotted trace) and HEKgloCX3CR1 clone after addition of $0.5 \mu \mathrm{M}$ FSK. Luminescence reading began 3 minutes before addition of FSK (thick arrow). After $30 \mathrm{~min}$ of pre-stimulation (thin arrow), a vehicle (black trace), $100 \mathrm{nM}$ CCL2 (blue trace), $100 \mathrm{nM} \mathrm{CX3CL1} \mathrm{(red} \mathrm{trace),} \mathrm{or} 100 \mathrm{nM}$ CXCL12 (green trace) was added. (B) The signals observed in A with CX3CL1 (red trace), CXCL12 (green trace) or CCL2 (blue trace) treatment were subtracted from the signal obtained with the vehicle (black trace in A) and were expressed either as a luminescence unit (left ordinate) or as a percentage of the maximum control signal (right ordinate) versus time after CHK treatment. (C) Responses to 100 nM CX3CL1 were measured as in Figure 1A except that the CHK was added 10 min (blue trace), $30 \mathrm{~min}$ (red trace) or $60 \mathrm{~min}$ (green trace) after FSK. Responses were expressed as a percentage of the maximum signal for the two first (blue and red traces) and as a percentage of the signal gained just before CHK treatment $(60 \mathrm{~min}$ ) for the green one. (D) Responses to $100 \mathrm{nM}$ CX3CL1 were measured as in 1A after addition of $0.5 \mu \mathrm{M}$ FSK (red trace), $1 \mu \mathrm{M}$ FSK (black trace), $5 \mu \mathrm{M}$ FSK (blue trace) or $20 \mu \mathrm{M}$ FSK (green trace) and analyzed as in Figure $1 \mathrm{~B}$ as a percentage of the maximum control signal.

Figure 2. Dose dependence of the CX3CL1-induced CAMP-inhibitory response

(A) The responses to $0.3 \mathrm{nM}$ (black trace), $1 \mathrm{nM}$ (green trace), $2.5 \mathrm{nM}$ (red trace), and $5 \mathrm{nM}$ of CX3CL1 (blue trace) were measured and analyzed as in Figure 1 as a percentage of the maximum control signal. The response to $100 \mathrm{nM}$ CX3CL1 when cells were first pre-treated with pertussis toxin $(100 \mathrm{ng} / \mathrm{mL}, 4 \mathrm{~h})$ is reported as grey trace. (B) The maximum amplitude of the response to various CX3CL1 concentrations in the presence of $0.25 \mu \mathrm{M}$ FSK (black circles) or $1 \mu \mathrm{M}$ FSK (red circles) are reported. The dose-response curve was analyzed using PRISM 5 (GraphPad Software) and calculated an EC50 of $0.30 \mathrm{nM}(\operatorname{LogEC} 50=-0 ; 53 \pm 0.08)$ and $0.25 \mathrm{nM}(\operatorname{LogEC} 50=-0.60 \pm$ $0.08)$, respectively.

Figure 3. Luminescent signals and cAMP-inhibitory responses observed after sequential or simultaneous addition of CX3CL1 and CXCL12

(A) Luminescence signals measured in the HEKgloCX3CR1 clone after addition (grey arrow) of $0.5 \mu \mathrm{M}$ FSK. After $30 \mathrm{~min}, 50 \mathrm{nM}$ of CX3CL1 (bold black arrows), CXCL12 (empty arrows), or CCL2 (thin black arrows) was added. Another $30 \mathrm{~min}$ later, one of the CHKs was added at the same concentration (traces a-e). Alternatively, both $50 \mathrm{nM}$ CX3CL1 and $50 \mathrm{nM}$ CXCL12 were simultaneously added $30 \mathrm{~min}$ after FSK pre-stimulation (trace f). (B) The CX3CL1 responses subsequent to CXCL12 or CCL2 addition observed in A were analyzed as in Figure $1 \mathrm{~B}$ and reported as a percentage of the signal gained just before CX3CL1 treatment. (C) The CXCL12 responses subsequent to CX3CL1 or CCL2 addition observed in A were analyzed as in Figure 1B and reported as a percentage of the signal gained just before CXCL12 treatment. The traces in B and $\mathrm{C}$ have the same color codes as in A.

Figure 4. The CX3CL1-induced CAMP-inhibitory response is dependent on surface CX3CR1 receptors

(A) The response to $5 \mathrm{nM} \mathrm{CX3CL1}$ in the presence of $0.5 \mu \mathrm{M}$ FSK (trace a) is shown as measured in Figure 1B. Simultaneously to CX3CL1 (trace b) or 8 min (trace c), 15 min (trace d), $30 \mathrm{~min}$ (trace e), or $60 \mathrm{~min}$ (trace $\mathrm{f}$ ) after CX3CL1 addition, $300 \mathrm{nM} \mathrm{F1}$ was added (red arrows). (B) CX3CR1 expression on the surface of the HEKgloCX3CR1 clone cells was analyzed using flow cytometry at various time points $(10,15,30,60$, and $90 \mathrm{~min})$ after addition of $50 \mathrm{nM}$ CX3CL1 without (black circles) or with 30 min treatment with $120 \mu \mathrm{M}$ dynasore (blue circles). At each time point, the data represent the ratio of the mean fluorescence intensity obtained in the presence of CX3CL1 to the mean fluorescence intensity obtained in the absence of CX3CL1. The data were 
analyzed using PRISM 5 (GraphPad Software) and gave a characteristic time of 14 and 579 min in the absence and presence of dynasore, respectively. (C) The responses to $50 \mathrm{nM} \mathrm{CX3CL1}$ observed in the absence (black trace) or presence (blue trace) of pre-dynasore treatment $(30 \mathrm{~min}, 120 \mu \mathrm{M})$ in the presence of $0.5 \mu \mathrm{M}$ FSK were measureed as in Figure 1B.

Figure 5. Model used to fit the CHK-induced cAMP-inhibitory responses

(A) Variation of the $\alpha$ parameter (rate of synthesis of cellular cAMP) at the time $t_{0}$ of FSK addition. $\alpha$ instantaneously steps from an initial value $\alpha_{i}$ to a final value $\alpha_{0}$. (B) Variation of the $\alpha$ parameter at the time $t_{1}$ of $\mathrm{CHK}$ addition (hence called $\alpha_{\mathrm{CHK}}$ ), as given by equation (3) (see text). (C) Variation of $\mathrm{C}$, the cellular cAMP concentration, at the time $\mathrm{t}_{0}$ of FSK addition derived from equation (2) (see text). (D) Variation of $\mathrm{C}$ at the time $\mathrm{t}_{1}$ of $\mathrm{CHK}$ addition (hence called $\mathrm{C}_{\mathrm{CHK}}$ ), derived from equation (4) (see text).

Figure 6. Fits of the FSK signal and CHK-induced cAMP-inhibitory response

(A) Luminescence signal obtained after addition of $0.5 \mu \mathrm{M}$ FSK (from Figure 1A) fitted either with equation (2) from model A (red trace) or equation (7) from model B (blue trace) (Supplemental Text S7). The parameters used were $C_{i}=241, t_{0}=13.0 \mathrm{~min}$, and $\tau_{B}=7.2$ min for both models. For model A, $\alpha_{0}=64.2$ luminescence unit.min ${ }^{-1}$. For model B, $\alpha_{0}=422.1$ luminescence unit.min ${ }^{-1}, t_{d}=$ $6.08 \mathrm{~min}$, and $\mathrm{k}_{\mathrm{d}}=1.06$. (B) CX3CL1 response from Figure 1B (CX3CL1 added 30 min after FSK pre-stimulation) fitted with equation (5) from model A (red trace) or with equation (9) from model $\mathrm{B}$ (blue trace) (Supplemental Text S7). The same parameters from Figure 6A were used, adding $\mathrm{p}=$ 0.50 and $\tau_{1}=40.6 \mathrm{~min}$ for model $\mathrm{A}$ and $\mathrm{p}=0.48$ and $\tau_{1}=293 \mathrm{~min}$ for model B. (C) CX3CL1 responses from Figure 2A, where CX3CL1 was added at $0.3,1,2.5$, and $5 \mathrm{nM}$, were fitted with equation (5) from model $\mathrm{A}$. The parameters used were $\tau_{\mathrm{B}}=7.6 \mathrm{~min}$ and $\tau_{1}=77.0 \mathrm{~min}$ for all traces. The parameter $p$, representing the amplitude of the $\alpha_{\mathrm{CHK}}$ step (Figure 5B), equals 0.85, 0.79, 0.74, and 0.65 for the respective fits. (D) CX3CL1 responses from Figure S4, observed in the presence or absence of IBMX, were fitted with equation (5) (orange and red traces, respectively). The parameters used were $p=0.61$ and $\tau_{1}=47.8$ for both traces. $\tau_{B}=4.7$ and 10.8 for the red and orange fits, respectively. (E) CX3CL1 responses from Figure 4C, observed in the presence or absence of dynasore pretreatment, were fitted with equation (5) (orange and red traces, respectively). The parameters used were $\tau_{B}=3.6$ for both traces. $\tau_{1}=46.0$ and $p=0.63$ for the red fit and $\tau_{1}=250.0$ and $p=0.69$ for the orange fit. All fits were done using Gnuplot v4.4. 